\title{
Les discours institutionnels du nucléaire. Stratégies discursives d'euphorisation
}

In: Mots, juin 1994, N³9. pp. 53-68.

Resumen

LOS DISCURSOS INSTITUCIONALES DEL NUCLEAR: ESTRATEGIAS DISCURSIVAS DE EUFORIZACION ¿ Cuál es la imagen que los responsables de la producción nuclear desean ver imponerse hoy en dia ? En una perspectiva de analisis del discurso, el examen de folletos informativos que emanan de grandes empresas nucleares en dirección del público, revela una demarcha toda inclinada hacia la euforización.

\section{Résumé}

LES DISCOURS INSTITUTIONNELS DU NUCLEAIRE: STRATEGIES DISCURSIVES D'EUPHORISATION Quelle est I'image que les responsables de la production nucléaire souhaitent voir s'imposer aujourd'hui ? Dans une perspective d'analyse de discours, l'examen des brochures informatives, qui émanent des grandes entreprises du nucléaire en direction du public, révèle une démarche tout entière tournée vers l'euphorisation.

Citer ce document / Cite this document :

Delavigne Valérie. Les discours institutionnels du nucléaire. Stratégies discursives d'euphorisation. In: Mots, juin $1994, N^{\circ} 39$. pp. 53-68.

doi : $10.3406 /$ mots. 1994.1886

http://www.persee.fr/web/revues/home/prescript/article/mots_0243-6450_1994_num_39_1_1886 
Les réactions en chaine les moins bien connues ne sont pas celles de la fission nucléaire, mais celles des transformations sociales. (Jean-Marc Lévy-Leblond,

L'esprit de sel, Paris, Le Seuil, 1984, p. 258)

\title{
Les discours institutionnels du nucléaire stratégies discursives d'euphorisation ${ }^{\circ}$
}

\begin{abstract}
Dans la polyphonie discursive à l'origine de l'objet de connaissance nucléaire civil, il y a de toute évidence une voix à ne pas oublier: celle des responsables de la production. Certes, restons consciente du fait que le public est toujours pris entre deux feux : le discours «rose» des producteurs et le discours politisé des médias et des partis. Néanmoins, il est intéressant d'examiner les discours des responsables en direction du public, dans la mesure où ce sont eux qui deviendront thème de discours médiatisés.

Le nucléaire civil est un enjeu civique important et, en tant que tel, un objet politique, dans le sens positif (le développement technologique, le progrès), ou négatif (la pollution, le danger, l'homme jouant à l'apprenti-sorcier). Mais le phénomène central dont s'emparent politiques et médias, ce n'est pas le nucléaire en tant que procédé de production, ensemble technologique, mis en

- Cet article est dédié à la mémoire de Louis Guespin, disparu le 18 décembre 1993. Fondateur du Groupe de recherches en terminologie (URA 1164, CNRS), il a été l'initiateur de cette étude et a toujours soutenu nos travaux avec la chaleur qui lui était coutumière.
\end{abstract}


œuvre par la science. Ce sont les discours-origines, discours scientifiques, rapports techniques, et, entre autres, les discours émanant des producteurs de l'énergie nucléaire en direction du public.

Il nous parait indispensable de considérer ce type d'énoncés au sein de la pluralité des discours-sources, et de sonder leur opérativité sur le public. En quoi contribuent-ils à former la conscience des destinataires ? Comment interviennent-ils : en simple composante, de façon complémentaire ou contradictoire par rapport aux discours naïfs, aux discours des médias, aux discours politiques, favorables ou hostiles ? Manquer d'étudier cet élément, c'est s'exposer à moins bien comprendre le procès de politisation du lexique du nucléaire civil.

Ayant mené autour de Paluel, commune normande qui abrite une centrale nucléaire, un début d'enquêtes orales sur l'investissement sémantique des termes par les premiers intéressés, les riverains, nous avons fait le constat, banal, de l'oscillation entre sérénité et inquiétude, et d'une attente explicitée du public d'une information disponible, claire et fiable ${ }^{1}$. Nous nous sommes donc polarisée sur la reformulation par les grandes entreprises du nucléaire - EDF, CEA, ANDRA, COGEMA - du discours scientifique et technique en direction du public. Notre attention se focalise donc sur des textes à la fois informatifs et quelque peu lénifiants, un type de discours assez répandu en définitive : la vulgarisation «en rose ».

\section{Des discours « roses »}

Le type de brochures dont nous nous proposons de démonter les mécanismes sont disponibles, soit sur les sites nucléaires, soit dans les agences qui dépendent de l'organisme concerné. Ces plaquettes évoquent un certain nombre d'aspects de l'énergie nucléaire. Nous interroger sur la forme qu'ils prennent doit mettre à jour, par une analyse des fonctionnements discursifs et sémiotiques, les options proposées aux destinataires.

Il s'agit de ne pas se limiter aux seuls aspects linguistiques de ses brochures. Comment en effet ne pas prendre en compte dans l'analyse des données sur l'énergie nucléaire, les images, au sens

1. Valérie Delavigne, Louis Guespin, «Nucléaire: risque et sécurité. Une recherche en socioterminologie », Terminologies nouvelles (Bruxelles), 8, 1992, p. 1925. 
propre du terme, qui circulent et cohabitent avec ces textes, pour finir par habiter les discours, l'ensemble de ce que Genette appelle la paratextualité, c'est-à-dire la relation d'un texte à son entourage (titres, sous-titres, illustrations) ? Le texte, sa mise en forme et les illustrations forment un tout insécable. L'ensemble des « unités périphériques » (croquis, photographies, commentaires, encadrés, accroches, etc.) ${ }^{l}$ est porteur de signification et concourt, directement ou non, à la production du sens.

Ainsi l'analyse ne saurait négliger les codes non linguistiques déployés, comme par exemple les couleurs (le vert, le bleu, le blanc dans la publicité télévisuelle de la COGEMA ${ }^{2}$, qui joue de manière ostensible sur le fantasme naturaliste) ou le graphisme, qui forgent une certaine image du nucléaire. Une présentation attrayante sur papier glacé, une typographie aérée, agrémentée de photos en couleur et de schémas simples et colorés contribuent à créer une impression d'ensemble, claire et lisse, impression que vient d'ailleurs confirmer le texte.

Considérons dans un premier temps les brochures EDF. Leur présentation est homogène. Quatre pages sont organisées de façon identique, séparées en deux colonnes: à gauche, des textes; à droite, des schémas, courbes ou dessins qui illustrent la colonne de gauche. Deux niveaux de lecture sont possibles. Les sous-titres et les schémas proposent une première lecture rapide, en délaissant le texte. Celui-ci peut faire l'objet d'une lecture plus approfondie. Ces textes ne peuvent être coupés des choix qu'ils autorisent et de leur mode de présentation.

\section{La légitimation économique}

Un des principaux arguments en faveur de l'énergie nucléaire est sa rentabilité par rapport à d'autres formes d'énergie. Une brochure est consacrée exclusivement à l' ${ }^{\prime}$ économie du nucléaire » ${ }^{3}$. Expli-

1. André Jean Petroff, «Sémiologie de la reformulation dans le discours scientifique et technique », Langue française, 64, 1984, p. 54.

2. La COGEMA, exploitant nucléaire au même titre que le CEA ou EDF, a mené une campagne publicitaire intitulée "COGEMA, la matière première du nucléaire". Cette campagne, destinée à présenter le groupe, a couvert à la fois la presse, la télévision et la radio.

3. EDF, L'économie du nucléaire (Eco. Nuc.), Clamart, Direction de l'équipement, EDF, 1989. 
cation de cette économie ou légitimation? C'est une frêle frontière, semble-t-il, qui sépare les deux.

La brochure commence par décrire les conditions d'émergence de la nouvelle politique énergétique instituée dans les années 1970 et qui va permettre au nucléaire de s'imposer : c'est ainsi que la crise pétrolière contribue à faire de la France «la deuxième puissance nucléaire du monde ». Le texte s'élabore ensuite sur les thèmes suivants : «Le programme électronucléaire a /.../ permis à la France de réduire sa dépendance énergétique, d'améliorer sa balance commerciale, de créer des emplois et de bénéficier d'une électricité à prix réduit »(Eco.Nuc.).

Indépendance énergétique, dont le taux a doublé en dix ans «grâce au développement de l'énergie nucléaire » et qui fait de celle-ci une énergie rentable; impulsion donnée au commerce extérieur par une contribution aux exportations ; création d'emplois ; conséquences financières de l'implantation d'un site nucléaire, non négligeables et bénéfiques au niveau de l'économie régionale (le montant des taxes versées à la commune et à la région est explicitement donné); baisse du prix du kilowattheure : chaque argument est présenté chiffres à l'appui, voire image à l'appui. Ainsi peut-on voir la photographie d'une école, ou encore une courbe de la facture énergétique française en chute libre. Chacun de ces facteurs vient en synergie contribuer à légitimer le choix du nucléaire.

Des repères temporels insistent sur la précocité de l'engagement français dans l'exploitation de cette énergie. Le fait que la France s'engouffre dans l'aventure nucléaire, en réponse à la crise pétrolière, est présenté comme un acte extrêmement judicieux puisque, «parallèlement au développement du nucléaire, l'électricité a pris une place de plus en plus importante dans les utilisations domestiques, industrielles et tertiaires »(Eco. Nuc.).

L'énergie nucléaire répond donc à la boulimie énergétique que connaissent les pays industrialisés depuis la seconde guerre mondiale. L'augmentation du prix du pétrole fait du combustible nucléaire «la source de production d'électricité la plus rentable». Ainsi la France est-elle présentée comme ayant su faire face aux aléas économiques de façon adéquate et satisfaisante.

Notons que c'est la France, en tant qu'instance abstraite, qui a le premier rôle: dès la première ligne de la brochure, elle entre en jeu et est posée comme acteur principal. L'orgueil national joue dans l'argumentation un rôle non négligeable: «Forte des connaissances techniques et scientifiques et de l'expérience industrielle 
acquise pendant près de trente ans, la France a lancé un ambitieux programme de construction électronucléaire, et développé parallèlement une industrie du cycle combustible adaptée aux besoins de ce programme » (Eco.Nuc.). "La France est l'un des rares pays au monde à disposer sur son territoire des installations nécessaires à la maitrise de toutes les opérations du cycle combustibles nucléaire ${ }^{1}$.» L'apparition récurrente de la France présente le choix du nucléaire comme étant le fait d'une instance nationale, supérieure, transcendante, dont EDF est exclue, dépositaire non de la décision, mais seulement de sa mise en œuvre. Cette itération est en outre liée à l'emploi d'un nous qui réfère aux Français et invite le lecteur à se rallier aux faits présentés.

« Ambitieux programme », « industrie adaptée », « un des rares pays au monde », le fait que la France soit devenue la deuxième puissance nucléaire du monde est appuyé par l'utilisation de tournures mélioratives, véhiculant des valeurs qui sont senties comme positives. Le terme même de puissance fait référence à l'idéologie d'une France forte, économiquement et techniquement. Il réactive un certain nationalisme en renouant avec le paradigme puissance coloniale, puissance économique...

Outre le fait que cette industrie témoigne de la puissance économique de la France, le nucléaire incarne la notion de progrès. Cette notion se retrouve sous les mots de Jean Bergougnoux, directeur général d'EDF, qui définit le nucléaire comme «une aventure industrielle sans précédent et sans équivalent dans le monde ${ }^{2}$. La représentation du nucléaire qui circule dans le discours officiel valorise le progrès technique que concrétise cette industrie et exprime la grandeur que doit en retirer la France.

L'ensemble des arguments économiques, apparemment indiscutables, tend à la légitimation du nucléaire et veut démontrer la pertinence du choix énergétique effectué. Les problèmes de sureté n'apparaissent pas dans cette présentation économique : une plaquette leur est entièrement consacrée.

1. EDF, Le cycle du combustible nucléaire, Clamart, Direction de l'équipement, EDF, 1989.

2. Jean Bergougnoux, Discours d'ouverture, à paraitre dans Le nucléaire du futur, Actes du Colloque ADAPes (Association des Amis de Passage), Paris, 29 septembre 1993. 


\section{Le nucléaire et l'environnement}

Les avantages attribués au nucléaire en termes d'impact sur l'environnement sont de deux types. D'un côté, le nucléaire contribuerait à réduire l'« effet de serre » en limitant l'utilisation des énergies fossiles qui émettent une grande quantité de dioxyde de carbone, générateur du phénomène : l'exploitation de l'énergie nucléaire permettrait donc de sauvegarder l'environnement. La production d'autres polluants comme, par exemple, le dioxyde de soufre, en serait également diminuée. D'autre part, l'utilisation du nucléaire contribuerait à préserver les ressources planétaires de combustibles fossiles.

Examinons la brochure publiée par EDF, Centrales nucléaires et environnement ${ }^{1}$. Le terme environnement est pris dans un sens très large puisque le terme intègre aussi bien les influences diverses et variées qui peuvent intervenir au niveau de l'eau ou de l'air, que les nuisances sonores ou esthétiques. Une des caractéristiques de cette présentation du nucléaire est de rapprocher l'énergie nucléaire d'autres types d'industries moins spécifiques (cf. supra en matière de déchets nucléaires).

«L'implantation d'une centrale nucléaire, comme celle de n'importe quel ouvrage industriel, influence nécessairement l'environnement. » (Cen. Nuc.)

Afin de gommer la spécificité des sites nucléaires et les transformations que ceux-ci peuvent faire subir à l'environnement, il semble nécessaire aux rédacteurs de montrer d'entrée de jeu que les influences, éventuelles et contrôlées, de l'implantation d'une centrale sur l'environnement ne sont pas seulement le fait de l'industrie nucléaire. Cette tentative de rapprocher les industries nucléaires de celles dites « classiques » se retrouve très fréquemment dans les brochures des différents organismes producteurs.

La plaquette souligne le fait que des études paysagères sont mises en cuvre afin d'évaluer l'impact paysager des sites nucléaires: «C'est ainsi par exemple que les réfrigérants atmosphériques de la centrale de Saint-Laurent ont été conçus pour échapper à la vue depuis la terrasse du Château de Chambord située à $15 \mathrm{~km}$, en dépit de la perte de puissance qui en résulte pour la centrale » (Cen. Nuc.). Notons la formule concessive en fin de phrase qui souligne les efforts effectués par les concepteurs.

1. EDF, Centrales nucléaires et environnement (Cen. Nuc.), Clamart, Direction de l'équipement, EDF, 1990. 
En dehors de l'influence esthétique qui reste tributaire du choix du site d'implantation, l'influence sur l'environnement est, selon ces brochures, quasi nulle; là encore, le discours se fait «rose ». «Après la mise en service de quelques 56 réacteurs de 900 et $1300 \mathrm{MW}$ et plus de 12 années d'exploitation des centrales, le bilan est le suivant : aucun effet majeur des installations nucléaires sur l'environnement naturel n'a été mis en évidence; les effets thermiques sur la vie aquatique et les effets climatiques des rejets atmosphériques, notamment, se sont avérés inférieurs aux prévisions et négligeables dans l'ensemble »(Cen. Nuc.).

« Aucun effet majeur », " effets inférieurs aux prévisions », « effets négligeables dans l'ensemble », «aucun effet significatif», "prise de toutes les dispositions nécessaires » afin que « la centrale puisse fonctionner en totale compatibilité avec le milieu dans lequel elle s'insère »: tout n'est-il pas pour le mieux dans le meilleur des mondes? Le texte est parsemé de mots destinés à rassurer. La récurrence du terme effet, liée à une négation ou à une minimisation, amplifie l'idée qu'aucune inquiétude n'est légitime. Au fil du texte, l'éventualité de chaque problème est évacuée ; rien n'est à craindre. Tout est soumis à une étroite surveillance, à des contrôles. Aucune défaillance n'est envisagée. Il n'en faudrait guère plus pour que le lecteur soit conduit à imaginer qu'elle n'est pas envisageable.

Loin d'être néfastes, les conséquences engendrées par l'exploitation de l'énergie nucléaire sont montrées comme étant bénéfiques pour l'environnement, puisque «le développement des centrales nucléaires a permis de réduire considérablement l'utilisation des centrales au fuel et au charbon, et donc les rejets d'oxyde de souffre, d'azote et de poussière. Depuis 1980 , les rejets polluants dus à ces centrales ont diminués de plus de $60 \%$ »(Cen. Nuc.).

L'hypothèse d'un accident est certes évoquée, non pour envisager son impact éventuel sur l'environnement, mais pour mettre en évidence le fait que «dans tous les cas, des dispositions sont prévues pour limiter les effets sur l'environnement à un niveau très faible » (Cen. Nuc.).

\section{L'impossible accident}

Si l'amélioration de la sureté reste dans les objectifs affirmés de la stratégie d'EDF, le discours des responsables dans ces brochures fait néanmoins preuve d'une quiétude à toute épreuve et d'un 
optimisme sans partage quant à la fiabilité du parc électronucléaire français.

La question de la sureté des installations nucléaires occidentales n'est jamais remise en cause dans les discours officiels des responsables. Les équipements sont d'une "sureté irréprochable » et ont bénéficié du «retour d'expérience » né de l'effet de «standardisation » (la France s'est équipée en centrales de même type). N'apparait nulle évocation d'éventuels défauts de conception qui pourraient surgir et se retrouveraient du fait de cette standardisation d'un bout à l'autre du parc.

Au niveau humain, l'erreur est prise en compte : ce qui apparait sous le syntagme culture de sureté bénéficie de la transparence, et assure une sureté optimisée. Les responsables se félicitent que les 25.000 années réacteurs se soient passées sans incident, même si une alerte grave (Three Miles Island) et une catastrophe (Tchernobyl) ont jeté un doute - immotivé ? - au niveau du public sur une sureté exemplaire. Si l'inquiétude des personnes compétentes ne se cache pas en ce qui concerne les installations nucléaires de l'Est, aucune remise en cause n'est apparemment effectuée quant aux installations occidentales. L'irruption dans les médias du nom des filières RBMK (à laquelle appartient la centrale de Tchernobyl), dont la caractéristique principale est de ne pas posséder d'enceinte de confinement, permet de focaliser sur ce seul type de centrale le risque d'accident. Les énoncés le soulignent avec vigueur. Par voie de conséquence, nul risque qu'un tel accident se produise en France : «Une affaire Tchernobyl en France, c'est impossible »'. Des différences notables séparent les deux filières : le schéma des deux types de réacteurs le montre clairement. Cependant, "même si la technologie mise en œuvre à Tchernobyl n'a que peu de points communs avec celle utilisée dans les centrales EDF, on doit toujours prendre en compte les enseignements d'un tel accident pour améliorer la sureté » (Cen. Sec.).

L'accident nucléaire est donc «hautement» ou «extrêmement peu probable ». Tout a été prévu : «Tous les scénarios envisageables ont été étudiés et sont connus » (Cen. Sec.), «toutes les hypothèses ont été envisagées » (Cen. Nuc.). «La situation ne peut pas se dégrader brusquement et les signes avant-coureurs de problèmes sont perceptibles longtemps à l'avance » (Cen. Sec.), «l'échec complet de toutes les mesures de sureté, et le percement de la

1. EDF, Centrales et sécurité (Cen. Sec.), Clamart, Direction de l'équipement, EDF, 1986, 6 p. 
cuve par la fusion du cœur ont même été imaginés" (Cen. Nuc.). L'ensemble des signes convoqués affirme une sureté maximale; nulle faille n'est envisageable. L'ensemble du texte est argumenté pour montrer que l'accident est d'une probabilité quasi nulle.

Le mot accident lui-même n'est utilisé qu'en étant dénié ou pour montrer que tout est fait pour qu'il ne puisse avoir lieu. L'affirmation de la priorité donnée à la mise en place de structures destinées à assurer la sûreté et la description de ces principes prennent le pas sur la manière dont un accident pourrait se produire : "La moitié du prix de revient de l'électricité produite dans une centrale nucléaire française correspond aux mesures de sécurité prises pour éviter les accidents quels qu'ils soient » $($ Cen. Sec.). On parle plus facilement dans ce type de discours d'incident que d'accident, le premier ayant, au niveau technique, une portée et des conséquences réduites par rapport au second.

Chaque terme a certes une définition technique bien précise! Mais dans un contexte linguistique où l'euphémisation est de rigueur, le mot d'incident fait trop visiblement office de litote. Les stratégies d'évitement du terme accident le font remplacer par problèmes ou situation préoccupante: « $\mathrm{Si}$ la situation devient préoccupante, le rempart de l'enceinte de confinement peut arrêter toutes fuites massives "(Cen. Sec.). Le maitre mot en matière de sureté est lâché : confinement.

Les conséquences dramatiques de l'accident de Tchernobyl sont liées à l'absence d'enceinte de confinement. Rempart, barrière, enceinte de confinement: les termes de sureté sont essentiellement sur le registre de l'enfermement. Il s'agit de contenir la radioactivité, d'éviter toute fuite. C'est donc a contrario que se profile ce qui pourrait constituer cet «impossible accident».

En fait, le mot accident est un mot vide de sens. Aucun moyen discursif ne permet au lecteur de se le représenter : fuite insidieuse ? fuite brutale et massive ? explosion ? Il est impossible à partir des éléments du texte de se faire une représentation de ces scénarios. L'accident est purement abstrait, inimaginable au sens propre du terme.

\section{Les déchets nucléaires}

Le problème des déchets est, selon les propres termes des responsables, le «sujet le plus controversé ». De fait, la gestion des déchets pose un "véritable problème de société " ${ }^{1}$ qui reste encore

1. ANDRA, La gestion des déchets radioactifs. Questions/Réponses, Fontenayaux-Roses, Direction de la communication, ANDRA, p. 11 et 20. 
en suspens. En affirmant que «préserver l'environnement relève de l'intérêt général "' ${ }^{1}$ l'ANDRA, organisme qui gère et stocke les déchets radioactifs, sous-entend que c'est également son propre intérêt, présupposant donc une sureté optimisée.

Dans un premier temps, la démarche discursive, mise en œuvre dans les brochures d'EDF et de l'ANDRA consacrées aux déchets radioactifs, tend vers une banalisation de ces déchets. Elles les replacent dans l'ensemble des déchets que génère toute industrie humaine : «Toute activité humaine produit des résidus. L'utilisation des propriétés radioactives de certains éléments, que ce soit pour la production d'électricité ou dans les domaines de la santé, de l'industrie ou de la recherche, ne fait pas exception à cette règle ${ }^{2}$.

Les brochures précisent que si les centrales nucléaires produisent $90 \%$ des déchets nucléaires, elles ne sont pas les seules sources de déchets radioactifs. Des photos d'utilisations médicales de la radioactivité viennent le rappeler.

Le contexte iconique et le contexte verbal peuvent conjuguer leurs sens, ce qui est généralement le but souhaité. Mais ils peuvent aussi se contredire. Le texte d'EDF (Déc. Rad.) insiste sur le faible volume des déchets produits (p. 2 et 4 ), tandis qu'une photo p. 3 présente un entassement de fûts dont la quantité ne semble pas négligeable: «En deux semaines, la ville de Paris doit évacuer un volume de déchets équivalent à celui qui aura été stocké sur le site de La Hague en 1991, soit $400000 \mathrm{~m}^{3}$ » (Déc. Rad.).

La comparaison des temps respectivement mis en jeu pour une quantité identique est frappante. L'ANDRA utilise une autre comparaison concernant les déchets les plus dangereux : ainsi « une piscine de jardin ${ }^{3}$ suffirait à contenir les déchets à vie longue produits chaque année. Il est à noter que le problème du démantèlement des centrales et de la quantité de déchets produits à cette occasion n'est évoqué dans aucune des brochures.

L'utilisation de la décroissance de la radioactivité en fonction du temps pour les déchets à vie courte ne porte certes pas à controverse. En revanche, la gestion des déchets à vie longue engendre un certain nombre de problèmes qui dépassent le cadre d'un organisme, dans la mesure où cette gestion utilise des chronologies vertigineuses,

1. ANDRA, brochure de présentation (sans titre), Fontenay-aux-Roses, ANDRA, 1993.

2. EDF, Les déchets radioactifs (Déc. rad.), Clamart, Direction de l'équipement, EDF, 1989.

3. ANDRA, La gestion des déchets radioactifs. Questions/Réponses, Fontenayaux-Roses, Direction de la communication, ANDRA, 1993, p. 6. 
à échelle non humaine, et engage les générations à venir. «L'objectif de la gestion des déchets radioactifs est d'assurer, pendant toute la durée nécessaire et en toutes circonstances, la protection des populations contre les risques radiologiques. Elle doit également préserver l'environnement et ne pas laisser aux générations futures le soin de trouver une solution pour les résidus produits par notre époque »(Déc. Rad.).

Les objectifs affirmés sont donc les suivants: protection de l'environnement et des populations contre toute pollution radioactive, et prise de responsabilités vis-à-vis des générations futures. L'ANDRA fait de l'appartenance des organismes concernés aux pouvoirs publics un gage de pérennité.

A la polémique suscitée par l'accident de Tchernobyl et au traumatisme qu'il constitue, les politiques répondent par la nécessité de la transparence. Cette nécessité s'institutionnalise tardivement (loi de décembre 1991). Les responsables répercutent cette volonté de transparence à tous les niveaux d'atteinte du public. Par exemple, elle s'affiche clairement dans des encarts de journaux qui paraissent dans les régions limitrophes aux centrales nucléaires : de façon régulière dans l'hebdomadaire haut-normand le Courrier Cauchois apparait une invite à visiter le Centre Nucléaire de Production d'Electricité de Paluel (abrégé en CNPE) et à demander des renseignements. Sur le site, devenu un lieu de tourisme très fréquenté, l'accueil du curieux est géré avec un très grand soin : visite guidée, conférence, exposition, brochures attendent le visiteur.

La démarche est identique à EDF et à l'ANDRA. La politique de transparence, de rigueur, invite chacun à «venir /.../ juger sur pièces dans les centres de stockages qui sont ouverts à tous ceux qui le souhaitent "'. C'est ainsi que «les (Commissions locales d'information) peuvent demander toute information qui leur parait nécessaire à l'ANDRA, aux organismes de contrôle, à des scientifiques indépendants, $y$ compris en faisant procéder à des contreexpertises $»^{2}$.

Affirmer ainsi l'ouverture à tous permet de générer la confiance. C'est, pragmatiquement, prolonger l'utilisation de termes qui affirment que les problèmes sont bien gérés, sous forme de tests réglementaires ou sévères, que la «sureté (est) maximale», que les « risques sont minimes», en tout cas «sans danger majeur », qu'ils

1. Ibid., p. 17.

2. Ibid., p. 23. 
ont un «impact négligeable », et que même en cas d'accident les «conséquences (seront) réduites».

A l'image des autres thèmes que nous avons abordés dans cette étude, la quiétude est de rigueur. Si le lecteur intègre pas à pas la démarche suivie, il est conduit à être totalement rassuré quant à la sureté et à la fiabilité des installations nucléaires. Il ne peut que suivre les dires des responsables selon lesquels le programme nucléaire est un «succès sur le plan des installations, de la sûreté, et sur le plan économique " ${ }^{1}$. Voici qui résume l'orientation générale des discours officiels : l'exploitation de l'énergie nucléaire est une parfaite réussite.

\section{Information, vulgarisation, publicité ?}

Quelle est la nature de ces discours émis par les responsables en direction du grand public? Il est tentant de rapprocher ces types d'énoncés des «discours de vulgarisation» dont ils présentent les caractéristiques formelles telles qu'elles ont pu être décrites par Daniel Jacobi ou Marie-Françoise Mortureux, par exemple ${ }^{2}$ : effacement de l'instance d'énonciation sous couvert de montrer une certaine neutralité à l'instar du discours scientifique; emploi de guillemets qui renvoient ici les termes mis ainsi en évidence non à un texte-source, explicitement cité, mais à une terminologie en usage; travail de réécriture de termes spécifiques et constructions diaphoriques qui posent une relation de substitution entre un mot et sa paraphrase, par le biais de parenthèses explicatives, de copules métalinguistiques qui lient le terme et sa glose («c'est-à-dire», « dit»), ou de verbes qui introduisent des définitions comme « on appelle », « on distingue », recours à l'analogie ou à la comparaison, iconographie nombreuse et variée. L'emploi de ces procédures descriptives qui exhibent un travail de reformulation, un usage autonymique des termes et l'importance du métalinguistique permettent à ces textes d'entrer dans la problématique de la vulgarisation. Divers modes de présentation des données sont en outre utilisés : brochures explicatives ou bandes dessinées, mais également panneaux, films, etc.

1. Pierre Bacher, directeur délégué à la Direction de l'équipement d'EDF. Communication a paraitre dans Le nucléaire du futur, op. cit.

2. Daniel Jacobi, Bernard Schiele (dir.), Vulgariser la science. Le procès de l'ignorance, Seyssel, Champ Vallon, 1988, 286 p. 
L'exploitation de l'énergie nucléaire civile est, comme toute technologie, un point d'interface entre la science et le profane. Sa spécificité consiste dans le fait que s'y greffent des enjeux nombreux dont les thèmes principaux gravitent autour des questions techniques de sûreté des installations, de stockage des déchets, et de considérations économiques et politiques. S'y ajoute une dimension mise en lumière par l'anthropologue Françoise Zonnabend : «Les opinions que l'on profère pour ou contre son développement sont rarement étayées par les seuls arguments techniques ou économiques; s'y mêle toujours une dimension symbolique qui leur donne un tour passionnel $»$.

La problématique de la vulgarisation est certes un point de vue pertinent, et l'aspect externe des brochures les fait entrer dans ce type de discours. Cependant, peut-on réellement considérer les actions d'information d'EDF, du CEA, de l'ANDRA ou de la COGEMA comme des activités vulgarisatrices ?

L'énergie nucléaire, sujet d'inquiétude collective, a bien une composante scientifique. Cependant, il ne s'agit pas de science à proprement parler, mais d'un ensemble de technologies mises en œuvre par des organismes dépendants des pouvoirs publics. Ceuxci ont en outre des engagements financiers qui ne peuvent aller de pair avec une information désintéressée, trait généralement attribué à la vulgarisation. Le lieu d'énonciation, dont on sait la pertinence, n'est pas le monde scientifique, mais une entité complexe faite d'hommes et de femmes qui possèdent une culture d'entreprise très forte.

Les discours des organismes nucléaires vers le public ont une fonction conative très prégnante. Ce sont des énoncés «orientés », qui cherchent à convaincre. Leur fonction première n'est pas de proposer des connaissances, mais de «faire passer» des messages du type : "L'énergie nucléaire n'est pas dangereuse », « la sécurité est assurée au maximum ». Ce trait les rapproche du discours publicitaire. Cette voie est d'ailleurs utilisée depuis 1991 par EDF et COGEMA, que ce soit par le biais de la télévision ou des journaux.

Le discours proposé dans les plaquettes de présentation est toujours sécurisant, jusque dans la relation des incidents. Notons d'ailleurs ici qu'à l'inverse les textes opposés à cette forme d'énergie sont généralement alarmistes et usent d'un langage catastrophiste.

1. Françoise Zonnabend, La presqu'ile au nucléaire, Paris, Odile Jacob, 1989. p. 181 . 
En ce sens, ces deux types de discours, en émettant une opinion, trahissent la vocation vulgarisatrice qui est de transmettre un contenu en tentant de respecter une certaine neutralité par rapport à ce contenu.

En second lieu, examinons un autre aspect de la vulgarisation. Une hypothèse courante sur ce type d'énoncés est de les considérer comme une « réénonciation de discours-sources, élaborés par et pour des "spécialistes", en discours seconds destinés à un large public ${ }^{\prime}$. D'aucuns parlent de traduction intralinguale, d'autres d'altération $^{2}$. Cette réénonciation met en cuvre des pratiques métadiscursives qui ont pour but de lever l'obstacle de terminologies opaques pour des non-spécialistes.

Or on ne peut trouver de «discours-sources» proprement dits pour l'énergie nucléaire, hormis pour les discours sur la fusion nucléaire, encore au stade de recherches expérimentales, mais il s'agit là d'une frange marginale de notre corpus et qui ne concerne pas directement l'exploitation de l'énergie nucléaire telle qu'elle est pratiquée actuellement.

Un autre trait distingue le type d'énoncés que nous examinons des textes couramment considérés comme vulgarisateurs. Comme le remarque Jean-Claude Beaune, la vulgarisation se fait «à l'ombre des techniques ${ }^{3}$. Il manque une dimension aux classifications effectuées en matière de vulgarisation: les discours techniques. Or on ne peut plus aujourd'hui séparer sciences et techniques, cellesci se posant comme interfaces concrètes entre la science et le profane. Est-ce la science ou la technique qui a envahi notre quotidien? Sont-ce des termes scientifiques ou techniques qui ont fait irruption au sein de notre langue de tous les jours? $L^{\prime}$ «aspartam», le « veau aux hormones», les « micro-ondes», les «plaques à induction», le «disque laser» appartiennent à quelle langue? Songeons aussi aux modes d'emploi, dont l'opacité du langage fait naitre de curieuses interrogations. Et la « radioactivité »? Langue technique? Langue scientifique ? Le constat de la continuité est déjà fait : c'est la raison pour laquelle Edgar Morin ${ }^{4}$ propose un macroconcept qui lie de façon interdépendante la science, la

1. Marie-Françoise Mortureux, «Paraphrase et métalangage dans le dialogue de vulgarisation », Langue française, 53, 1982, p. 3.

2. Jean Peytard, «Problématique de l'altération des discours: reformulation et transcodage », Langue française, 64, 1984, p. 17-28.

3. Jean-Claude Beaune, «La vulgarisation scientifique. L'ombre des techniques", dans D. Jacobi et B. Schiele (dir.), op. cit., p. 47-81.

4. Edgar Morin, Science avec conscience, Paris, Fayard, 1982, p. 69. 
technologie et l'industrie. Cependant, les classifications rejoignent la conscience commune en omettant de prendre en compte les textes techniques pour réserver aux seuls énoncés issus de la science le statut de vulgarisation. Doit-on aujourd'hui se contenter de ces classifications ? L'information scientifique et technique est disséminée dans les médias sous des formes diverses, et les techniques nucléaires n'échappent pas à cette règle.

Où placer alors les discours sur l'énergie nucléaire publiés par les responsables, ou ses détracteurs ? La notion de vulgarisation fait certes appel à une pluralité de pratiques. Cependant, faire entrer l'information nucléaire dans la problématique «vulgarisation scientifique et technique » ne permet pas de conclure. La spécificité des discours sur l'énergie nucléaire, par leur lieu d'énonciation, par leur visée, par leur absence de discours-sources, ne leur permet pas de se placer sous ce qu'on appelle d'ordinaire «vulgarisation » ou « popularisation ». Entre information et vulgarisation, la place de la diffusion des concepts et techniques de l'énergie nucléaire est mitoyenne. Il manque de fait un terme pour la qualifier.

Peut-on dans ce cas la rapprocher des discours publicitaires ? A première vue, la réponse est non. Cependant, il est légitime de récuser ce non quand on examine attentivement la publicité du type de celle que propose la COGEMA. Les mêmes formes discursives se retrouvent en effet à la fois dans la publicité et dans les plaquettes de présentation. Le choix de la voie publicitaire démontre en tout cas que c'est au-delà de la simple vulgarisation que souhaitent aller les responsables.

Si la majeure partie de l'objet analysé dans cet article reste au stade de l'écrit, ne négligeons pas qu'existe aussi une information « officielle » orale, conférences proposées sur les sites, réunions plus informelles dans certaines communes proches des centres nucléaires, visites commentées qui utilisent des supports audiovisuels dont la tonalité générale relève du même type d'euphorisation. 


\section{Résumé / Abstract / Compendio}

\section{LES DISCOURS INSTITUTIONNELS DU NUCLEAIRE : STRATEGIES DISCURSIVES D'EUPHORISATION}

Quelle est l'image que les responsables de la production nucléaire souhaitent voir s'imposer aujourd'hui ? Dans une perspective d'analyse de discours, l'examen des brochures informatives, qui émanent des grandes entreprises du nucléaire en direction du public, révèle une démarche tout entière tournée vers l'euphorisation.

Mots clés : énergie nucléaire, vulgarisation, analyse de discours

\section{INSTITUTIONAL DISCOURSES OF NUCLEAR POWER: DISCURSIVE STRATEGIES TO INDUCE EUPHORIA}

What is the image of nuclear power that its marketers are presently trying to impose on the public? Discourse analysis of informative brochures produced by the large nuclear power companies for dissemination to the general public reveals a marketing strategy wholly aimed at inducing euphoria.

Key words: nuclear energy, scientific popularization, discourse analysis

LOS DISCURSOS INSTITUCIONALES DEL NUCLEAR: ESTRATEGIAS DISCURSIVAS DE EUFORIZACION

¿Cuál es la imagen que los responsables de la producción nuclear desean ver imponerse hoy en día? En una perspectiva de análisis del discurso, el examen de folletos informativos que emanan de grandes empresas nucleares en dirección del público, revela una demarcha toda inclinada hacia la euforización.

Palabras claves : energía nuclear, vulgarización, análisis del discurso 